\title{
Effect of Gravitation on Water Migration in Granular Media
}

\author{
J.J. Milczarek, I. FiJAŁ-KirejczyK, J. ŻoŁA̧DeK, \\ M. Chojnowski and G. KowalczYK \\ Institute of Atomic Energy, Świerk, 05-400 Otwock, Poland
}

Dedicated to Professor Jerzy Janik on the occasion of his 80th birthday

\begin{abstract}
The results of the experiments on water migration in unsaturated granular media performed with dynamic neutron radiography technique are presented. It was found that the porosity of the medium did not determine the kinetics of the process in a marked way. The influence of gravity was found to be considerable for media like coarse sand and gravel, consisting of large grains. No effect of gravitation on the water migration in clay beds was observed. The results are discussed in terms of the classical WashburnBosanquet theory of adhesion driven motion of the liquid in a straight circular capillary under gravity. It was found that this theory provides only qualitative description of the water migration within granular media, and the viscous dissipation effects are greatly underestimated.
\end{abstract}

PACS numbers: 47.56.+r, 68.08.De, 81.70.- $\mathrm{q}$

\section{Introduction}

Water migration in porous media composed of individual grains is the main topic of soil science [1-3]. The rate of wetting and drying of soils is a crucial factor influencing the fertility and biological processes of soil [4]. On the other hand, the rate of migration of liquid moving under gravity is important for chemical engineering in processes like filtration [5]. Despite its centenarian history, the physics of fluid in porous media has drawn much attention in recent years [6] within the scaling laws approach.

The aim of the present work was to obtain experimental data on the spontaneous migration of water under gravity in unsaturated beds of natural simple soils as clay, sand and gravel, and in some prepared beds consisting of grains of given chemical composition and selected grain size. In our studies the neutron radiography technique was used, which has been proved to be perfect for determination of the wetting front motion and water distribution inside the rigid and 
granular porous media [7-12]. We hope that the results of our experiments with simple granular structures will help in planning future research on subtleties of water migration in granular media.

Recently, the spontaneous water imbibition in rigid porous systems was investigated systematically with various techniques such as gravimetric method, NMR, X-ray tomography and neutron radiography $[3,8,13-16]$ with no special attention paid to the influence of gravity. It was found that the motion of the wetting front in those systems complies at least approximately with the square root of time classical law [17]. However, even simple reasoning indicates that the presence of gravity modifies that dependence significantly producing the effect of maximum height of the imbibed region.

It is well known that the spontaneous imbibition of porous media with liquid is driven by the adhesion of liquid to pores' surfaces [1-3,13]. It is obvious that the liquid movement is temperature dependent because the interfacial energies as well as other characteristics of the liquid (density and viscosity) depend strongly upon the temperature. Some other factors like the thermal expansion of the grains or enhanced evaporation of liquid are probably less important. Since the ratio of the surface tension to the viscosity of water increases with temperature in the region from $5^{\circ} \mathrm{C}$ to $100^{\circ} \mathrm{C}$, the enhancement of the imbibition rate should be observed for higher temperatures. To test that expectation the water migration was observed for temperatures within $25-70^{\circ} \mathrm{C}$ region.

\section{Experiment and data analysis}

The experiments were performed on samples of three natural simple soil systems - clay, sand, and gravel. The material was collected from the natural beds in the central Poland region and prepared by sieving and drying. The grains composing clay and sand samples had on average diameters smaller than $0.1 \mathrm{~mm}$ and $1 \mathrm{~mm}$, respectively, with large spread of sizes. The diameters of the gravel grains were between 1 and $3 \mathrm{~mm}$, approximately. For comparison, three especially prepared granular materials made of quartz $\left(\mathrm{SiO}_{2}\right)$ and corundum $\left(\mathrm{Al}_{2} \mathrm{O}_{3}\right)$ were studied. The quartz sand were studied in two forms, one of fine grain bed, consisting of regular grains of diameter within the range $0.09-0.16 \mathrm{~mm}$, and the coarse grain bed, formed by grains of 1.0-1.2 mm diameter. The corundum beds consisted of irregularly shaped grains of approximately $0.05 \mathrm{~mm}$ size. The effective porosity of the beds was determined by gravimetric method and varied from 0.38 for coarse quartz to 0.5 for corundum (Table).

The materials for the samples were initially rinsed with deionized water for $24 \mathrm{~h}$ and then dried for $2-5$ weeks at $95^{\circ} \mathrm{C}$. The samples were the bed columns contained in aluminum cylindrical tubes of $7 \mathrm{~mm}$ inner diameter and $135 \mathrm{~mm}$ length and $1 \mathrm{~mm}$ thick wall. The samples were prepared approximately $1 \mathrm{~h}$ before start of each experimental run by filling the tubes with dry granular material. The fresh samples were prepared for each experimental run. During the experiment samples 
TABLE

The effective porosity, imbibition rate parameter at $30^{\circ} \mathrm{C}$ and effective capillary radius of the beds studied.

\begin{tabular}{c|c|c|c|c}
\hline \hline Material & $\begin{array}{c}\text { Effective } \\
\text { porosity }\end{array}$ & $\begin{array}{c}\text { Imbibition } \\
\text { rate parameter } \\
a\left[\mathrm{~mm} \mathrm{~s}^{-1 / 2}\right] \\
\text { at } 30^{\circ} \mathrm{C}\end{array}$ & $\begin{array}{c}\text { Effective capillary } \\
\text { radius } r_{a}[\mu \mathrm{m}] \\
\text { determined from } a\end{array}$ & $\begin{array}{c}\text { effective capillary } \\
\text { radius } r_{H}[\mathrm{~mm}] \\
\text { determined from } H\end{array}$ \\
\hline clay & 0.47 & 3 & 0.2 & - \\
natural sand & 0.38 & 3 & 0.2 & 0.36 \\
gravel & 0.48 & 5.3 & 0.63 & 0.15 \\
fine quartz & 0.44 & 8 & 1.45 & 0.1 \\
coarse quartz & 0.43 & 5.4 & 0.65 & 0.62 \\
corundum & 0.50 & 4.8 & 0.52 & -
\end{tabular}

were held in vertical position in special aluminum holder. The single layer of filter paper was closing the lower end of the sample whereas the upper end was left without cover. The water was introduced into the upper or lower container according to whether the water migration in the direction parallel or antiparallel to the gravitation force was studied (Fig. 1). The double-shielded thermal cell provided the isothermal environment conditions during the experiment. The environment temperature could be chosen from $25^{\circ} \mathrm{C}$ to $70^{\circ} \mathrm{C}$ and stabilized within $\pm 0.1^{\circ} \mathrm{C}$.

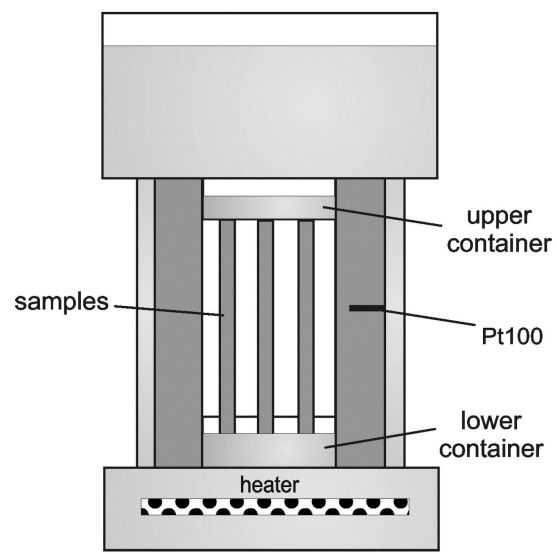

Fig. 1. The sketch of the experimental setup. Pt100 denotes the gauge used for stabilization of the temperature.

The water was introduced into appropriate (lower or upper) container approximately $90 \mathrm{~min}$ after closing the thermal cell when the deviations of the temperature gauge (Pt100 resistor, Fig.1) from the desired temperature were smaller than $0.1^{\circ} \mathrm{C}$. The moment of contact with water (when the water surface touches 
the appropriate end of the sample) was clearly identified on the computer screen during the experiment and defined precisely later by the inspection of the acquired images (Fig. 2). The wetting process was registered for the time necessary to saturate the samples with water, on the average at least for $20 \mathrm{~min}$ for the upward motion, at different temperatures chosen from the $25-70^{\circ} \mathrm{C}$ interval. For each of the recorded sequences of images the frames were taken every $3 \mathrm{~s}$ with the exposition time of $2.5 \mathrm{~s}$ or every $2 \mathrm{~s}$ with $1.6 \mathrm{~s}$ expositions.

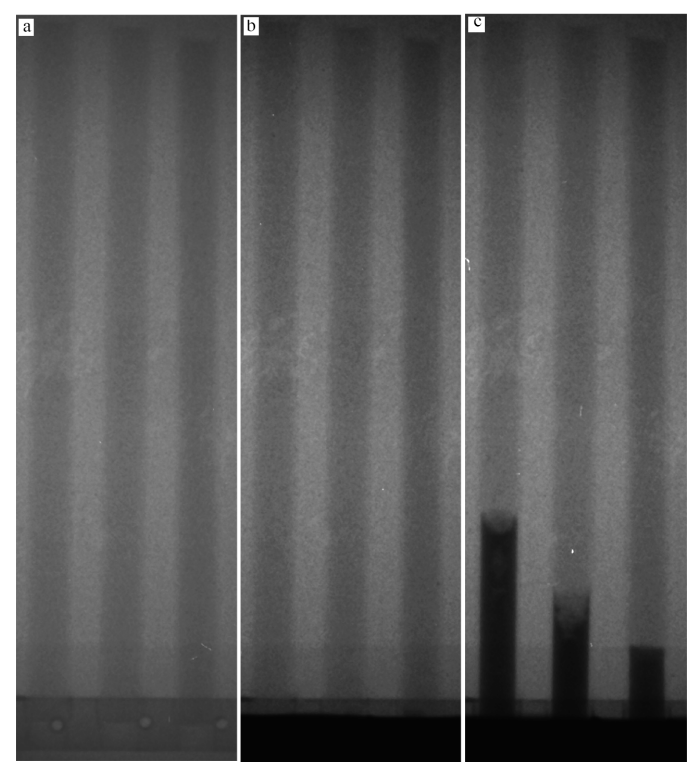

Fig. 2. Neutron images of water imbibition into three vertical samples, from the left: fine quartz, corundum, and clay. The pictures represent the system without water (a), with surface of water touching the lower ends of the samples (b), the imbibition process $29 \mathrm{~s}$ after moment of contact with water (c) - dark regions are imbibed with water.

The collected images were analyzed to determine the position of the wetting fronts propagating along the central lines of the samples. The front position defined as the distance $d$ of the edge of the dark region (Fig. 2c) from the appropriate end of the sample was determined from the recorded images with the accuracy of $0.2 \mathrm{~mm}$.

The dependence of the wetting front position $d$ on the time $t$ elapsed from the moment of contact with water is highly non-linear (Fig. 3). For the upward motion of the wetting front in natural sand, quartz beds, and gravel the saturation of the imbibed region height was observed (Fig. 3b-d).

The detailed analysis performed to check whether the power law

$$
d(t) \propto t^{\alpha}
$$

is applicable, revealed that only in the cases of clay and corundum the simple power law exponent $\alpha \approx 0.5$ can describe that time dependence. For the natural 

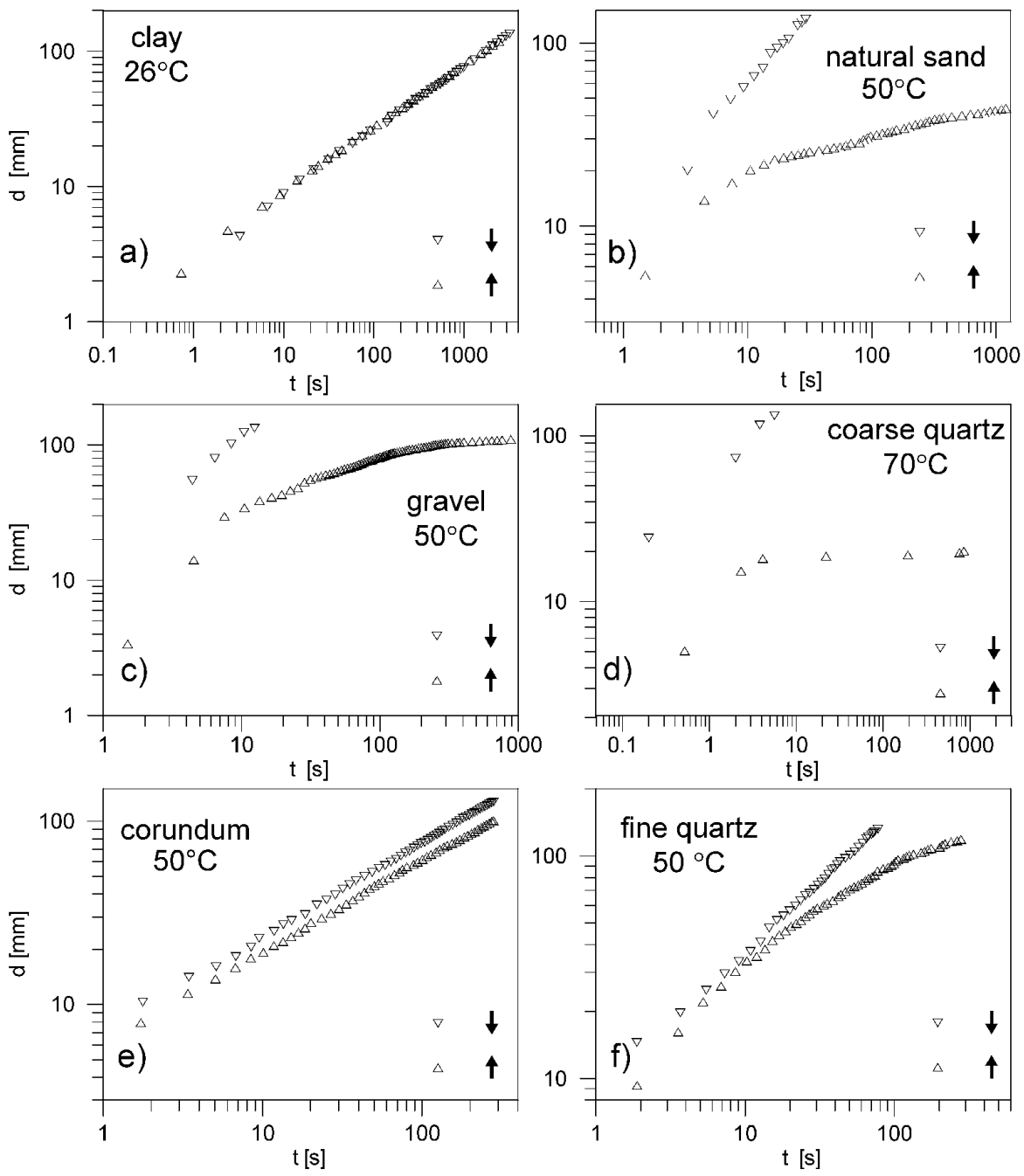

Fig. 3. Examples of the time dependence of the wetting front position in various media and temperatures: clay at $26^{\circ} \mathrm{C}$ (a), natural sand at $50^{\circ} \mathrm{C}(\mathrm{b})$, gravel at $50^{\circ} \mathrm{C}$ (c), coarse quartz at $50^{\circ} \mathrm{C}(\mathrm{d})$, corundum at $50^{\circ} \mathrm{C}(\mathrm{e})$, and fine quartz at $50^{\circ} \mathrm{C}$ (f). The vertical arrows, $\downarrow$ and $\uparrow$, indicate the direction of the wetting front motion, according and opposite to the gravity, respectively.

sand, fine and coarse quartz as well as gravel no single power law exponent can describe the dependence of $d$ on the time $t$. Even in the initial period of the process (first $25 \mathrm{~s}$ ) the data for natural sand and gravel are too scarce to estimate the fit parameters with reasonable accuracy.

The power law exponent determined from fitting the experimental points with formula (2) for clay sample is $0.503 \pm 0.005,0.474 \pm 0.007$, and $0.490 \pm 0.007$ 
for 30,40 , and $50^{\circ} \mathrm{C}$, respectively. For corundum the exponents are $0.51 \pm 0.01$, $0.50 \pm 0.01$, and $0.501 \pm 0.005$ for 25,50 , and $70^{\circ} \mathrm{C}$, respectively.

Attributing the departures of the exponent from the classical value to the incipient wetting processes we analyzed the wetting front motion at the early stages of the process with the generalized classical square root dependence

$$
d=a \sqrt{t}+b .
$$

The imbibition rate parameter $a$ at $30^{\circ} \mathrm{C}$ varies from approximately $3 \mathrm{~mm} \mathrm{~s}^{-1 / 2}$ for clay and natural sand to $8 \mathrm{~mm} \mathrm{~s}^{-1 / 2}$ for fine quartz with no systematic dependence on porosity (Table).

The imbibition rate parameter increases with the temperature: for clay it changes from $3 \mathrm{~mm} \mathrm{~s}^{-1 / 2}$ at $30^{\circ} \mathrm{C}$ to $4 \mathrm{~mm} \mathrm{~s}^{-1 / 2}$ at $50^{\circ} \mathrm{C}$, whereas for corundum it increases from $1.7 \mathrm{~mm} \mathrm{~s}^{-1 / 2}$ at $25^{\circ} \mathrm{C}$ to $2.7 \mathrm{~mm} \mathrm{~s}^{-1 / 2}$ at $70^{\circ} \mathrm{C}$.

\section{Discussion}

The simplest theoretical description of the wetting front motion in dry porous medium under capillary force is based on the Poiseuille-Hagen description of the viscous flow in circular capillary tube of radius $r$ supplemented with gravitational force [17-23]. Starting with those simplistic assumptions, forming the basis of the Washburn-Bosanquet approach [17-19], it can be shown that the time evolution of the distance $l$ of the meniscus from the liquid supplied end of the vertical capillary can be described by the equation

$$
\frac{\mathrm{d}^{2} w}{\mathrm{~d} \tau^{2}}+\frac{\mathrm{d} w}{\mathrm{~d} \tau}+\gamma \sqrt{w}=1,
$$

where $w$ is proportional to the square of $l$ :

$$
w=\frac{32 \eta^{2}}{\rho \sigma \cos \theta r^{3}} l^{2},
$$

the normalized time variable $\tau$ is proportional to the time $t$ :

$$
\tau=\frac{16 \eta}{\rho r^{2}} t,
$$

and parameter $\gamma$ is proportional to the gravity $g$ :

$$
\gamma=\frac{g \rho^{\frac{3}{2}} r^{\frac{5}{2}}}{8 \eta \sqrt{2 \sigma \cos \theta}} .
$$

The $\rho$ and $\eta$ variables are liquid's density and viscosity, respectively. $\sigma$ is the surface tension and $\theta$ the wetting angle of the capillary wall by the liquid.

When $\gamma>0$, the lower end of the capillary is immersed in the water container and liquid moves upwards, opposite to the gravity. For $\gamma<0$ the liquid supplied to the upper end of the capillary moves downwards, according to the gravity.

When the inertial component of the Newton equation is ignored, the analytical solution of the equation of motion is found and for $\gamma=0$ one finds the classical law

$$
l=a \sqrt{t} .
$$


According to classical theory [17] the effective capillary radius $r$, the ratio of the surface tension $\sigma$ and viscosity $\eta$ and the wetting angle $\theta$ determine the imbibition rate parameter $a$ :

$$
a=\sqrt{\frac{\sigma r \cos \theta}{2 \eta}} .
$$

Since no analytical solution of the full Eq. (3) is known, it was solved numerically in the normalized variables $(w, \tau)$ with Mathematica ${ }^{\circledR}$ for several values of $\gamma$. It is evident that the larger the absolute value of $\gamma$ the larger is difference between solutions for different signs of the gravitational parameter (Fig. 4).

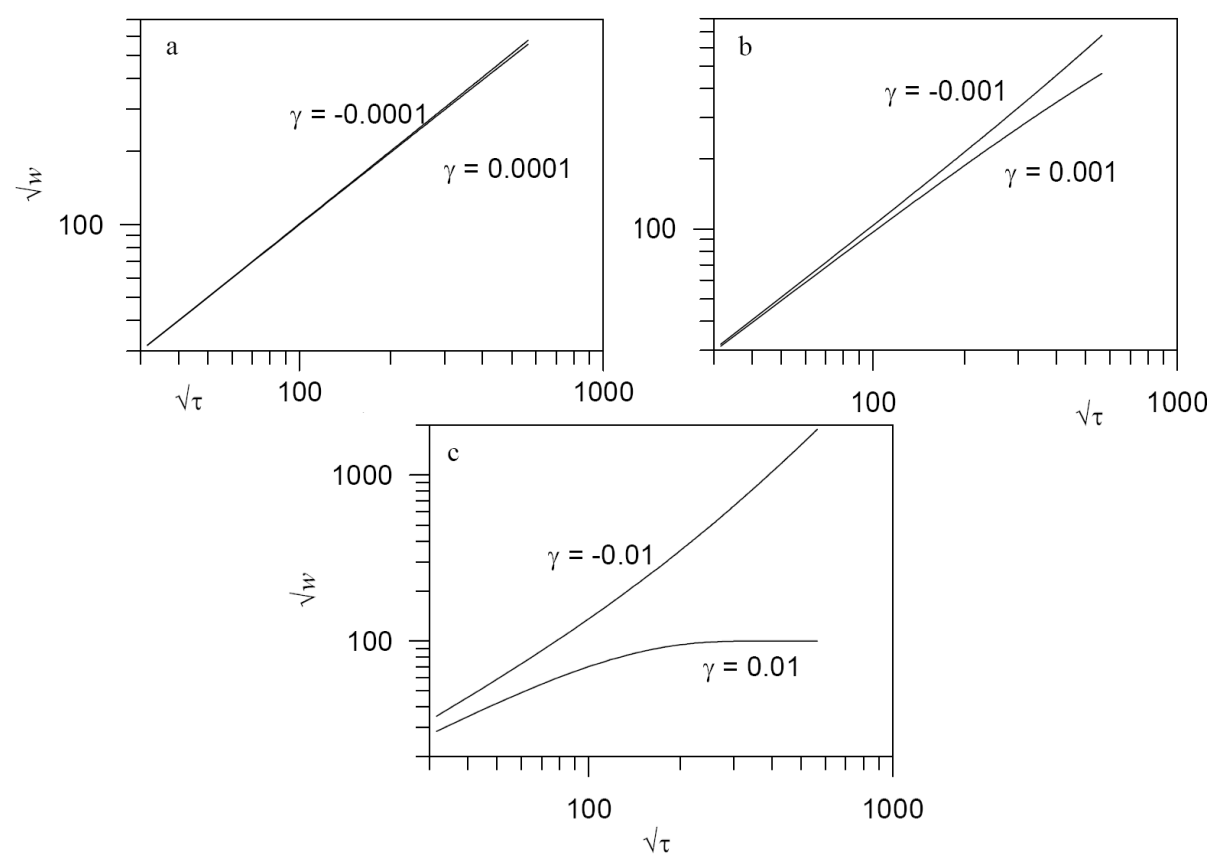

Fig. 4. Time dependence of the meniscus distance from the liquid immersed end of the vertical capillary plotted in $\log$-log scale for normalized $(w, \sqrt{\tau})$ variables for $|\gamma|=$ 0.0001 (a), 0.001 (b), and 0.01 (c). The negative and positive values of $\gamma$ correspond to the downward and upward motion of the liquid column, respectively.

In an attempt to model the imbibition process of the granular medium by the effective single capillary, the radius $r_{a}$ of such model tube was calculated from the rate parameter $a$ given by Eq. (8). The effective radius $r_{a}$ was calculated assuming zero wetting angle $\theta$ and the NIST data on the surface tension and viscosity of water [24]. The determined values of $r_{a}$ are within $0.2-1.5 \mu \mathrm{m}$ range (Table).

We should mention that the observed increase in the imbibition rate with increasing temperature does not match the expected dependence on temperature 
described by the square root of the surface tension to viscosity ratio and is approximately $15 \%$ smaller than the increase stipulated by Eq. (8).

The coarser grain beds - natural sand, fine and coarse quartz, gravel exhibit much more complicated behavior with tendency of reaching the maximum height $H$ at later stages of the process. Since the height saturation effect is due to the balance between capillary suction and gravity the effective radii of the straight capillary tubes representing the sample were calculated (Table) according to the formula

$$
r=\frac{2 \sigma \cos \theta}{H \rho g}
$$

with the same zero wetting angle assumption as before. The effective radius $r_{H}$ determined from the maximum height $H$ amounts to few tenths of millimeter, and is approximately few hundred times larger than the radius $r_{a}$ determined from initial imbibition rate. This difference indicates the very limitations of the classical theoretical description of imbibition in media where the influence of gravity is significant.
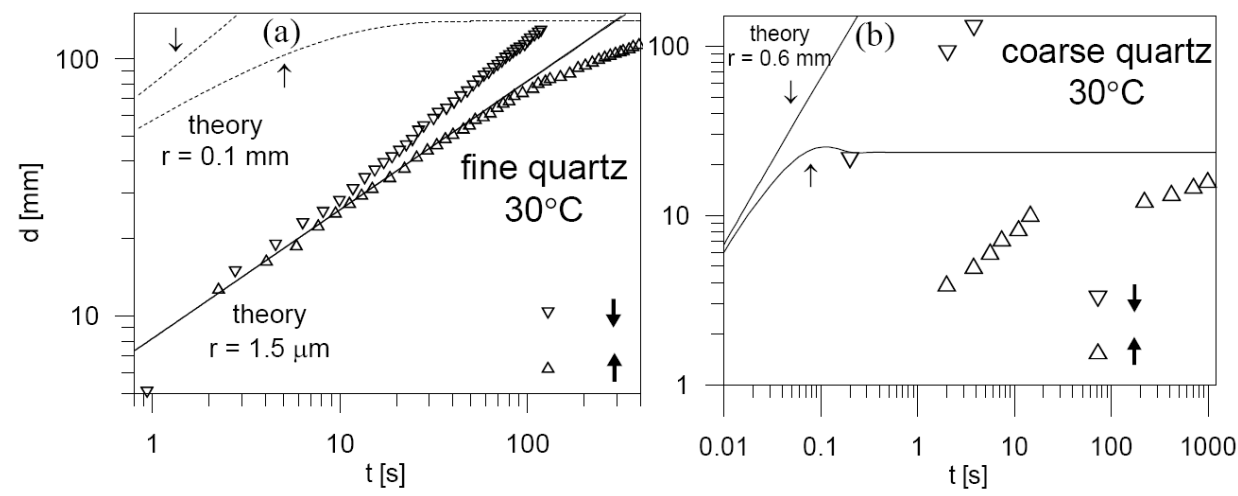

Fig. 5. Examples of the comparison of the Washburn-Bosanquet theory and the experimental data for fine (a) and coarse quartz (b). For fine quartz the theoretical curves are plotted for two different estimates of the effective capillary radius, one $(1.5 \mu \mathrm{m})$ yielded by initial imbibition rate and the other $(0.1 \mathrm{~mm})$ yielded by maximum height.

The detailed comparison of the theoretical model given by Eq. (3) to the experimental data reveals that theoretical curves calculated to fit the initial period of imbibition do not account properly for the effect of the gravity in the cases of natural sand and fine quartz (Fig. 5a). For these beds the theoretical curves hardly not split for two different directions of wetting front motion. Moreover, the theoretical dependence of water front position on time calculated with the effective radius determined from maximum height is at marked contrast with experimental data. For the beds consisting of large grains the theoretical curves, calculated with the maximum height radius estimation, reproduce only the asymptotic maximum 
height effect but yield extremely fast rise of water within initial period (Fig. 5b). This steep rise of theoretical curves as well as the damped oscillations of the wetting front predicted by the theory (Fig. 5b) indicate the underestimation of the energy dissipation at the pores' surfaces by unrealistic assumption of the smooth capillary walls.

\section{Summary}

We have demonstrated the applicability of neutron radiography for studies of the water imbibition in granular media. The main aim of obtaining data on the kinetics of the process and its dependence upon temperature was achieved.

The Washburn square root of time behavior for the wetting front motion was found approximately valid only for the media composed of finest grains i.e. clay and corundum. The increase in the imbibition rate with increasing temperature was noticed for these media but the effect of temperature should be studied more thoroughly in the considered temperature range. For natural sand and quartz beds as well as gravel the time dependence of the water front position deviates very strongly from the classical dependence. For most media the imbibition rate found for the initial period of the process is non-monotonic function of the temperature, the effect which may be attributed to details of incipient wetting of the grains forming the bed end surfaces. The imbibition rate estimations for the initial period would be more reliable if more frames were collected, which requires more luminous neutron source.

The presented results indicate that the capillarity is the main driving force of the imbibition in loose porous materials with fine grains. However, the gravity seems to have strong effect on the imbibition only in the beds composed of large grains. The maximum imbibition region height yields estimation of the effective pore size that is at variance with the estimates based on the imbibition rate which are reasonable for fine grained granular beds and rigid porous materials [10]. One should notice that the estimates based on the two methods differ by a factor of $\sim 100$. The difference should be attributed not only to differences in the media structure but mainly to the simplistic reasoning underlying the effective capillary tube picture.

Some of the irregularities observed in wetting front motion and water distribution profiles are certainly due to the axial non-uniformity (heterogeneity) of the samples. The reason may be the simple method of pouring the loose material into container tube. The heterogeneity of the samples may be caused by some kind of segregation of grains of different size as well as the grain arching effect. We hope that the irregularities will disappear for samples prepared by different methods yielding more homogeneous beds of granular systems composed of regular grains with purposefully tailored distribution of their diameters. 


\section{References}

[1] D. Hillel, Soil and Water. Physical Principles and processes, Academic Press, New York 1971.

[2] D. Kirkham, W.L. Powers, Advanced Soil Physics, Wiley-Interscience, New York 1972.

[3] A.W. Warrick, Soil Water Dynamics, Oxford University Press, Oxford 2003.

[4] F. Shaxson, R. Barber, Optimizing Soil Moisture for Plant Production. The Significance of Soil Porosity, FAO, Rome 2003.

[5] J.M. Coulson, J.F. Richardson, Chemical Engineering, Vol. 2, Pergamon Press, London 1956.

[6] M. Alava, M. Dubé, M. Rost, Adv. Phys. 53, 83 (2004).

[7] A. Czachor, A. El-Ghany el Abd, J.J. Milczarek, Acta Phys. Pol. A 102, 245 (2002).

[8] A. El-Ghany el Abd, J.J. Milczarek, J. Phys. D, Appl. Phys. 37, 2305 (2004).

[9] M.R. Deinert, J.-Y. Parlange, T. Steenhuis, J. Throop, K. Ünlü, K.B. Cady, J. Hydrol. 290, 192 (2004).

[10] A. El-Ghany el Abd, A. Czachor, J. J. Milczarek, J. Pogorzelski, IEEE Trans. Nucl. Sci. 52, 299 (2005).

[11] J.J. Milczarek, A. Czachor, A. El-Ghany el Abd, Z. Wiśniewski, Nucl. Instrum. Methods Phys. Res. A 542, 232 (2005).

[12] J. Ridgway, P.A.C. Gane, A. El-Ghany el Abd, A. Czachor, Transp. Porous Media 63, 503 (2006).

[13] F.A.L. Dullien, Porous Media: Fluid Transport and Pore Structure, Academic Press, New York 1979.

[14] J.J. Meyer, A.W. Warrick, Soil Sci. Soc. Am. J. 54, 1547 (1992).

[15] A. Guerini, D. Swartzendruber, Soil Sci. Soc. Am. J. 56, 335 (1992).

[16] A. Guerini, D. Swartzendruber, Soil Sci. Soc. Am. J. 62, 580 (1998).

[17] E.W. Washburn, Phys. Rev. 17, 273, 374 (1921).

[18] K. Rideal, Philos. Mag. Ser. 6 44, 1152 (1922).

[19] H. Bosanquet, Phil. Mag. Ser. 6 45, 525, 23 (19.)

[20] J. Szekely, A.W. Neumann, Y.K. Chuang, J. Colloid Interface Sci. 35, 273 (1971).

[21] K.S. Sorbie, Y.Z. Wu, S.R. McDougall, J. Colloid Interface Sci. 174, 289 (1995).

[22] T.L. Staples, D.G. Shaffer, Colloids Surf. A: Physicochem. Eng. Aspects 204, 239 (2002).

[23] H. Czachor, J. Hydrol. 328, 604 (2006).

[24] http://webbook.nist.gov/chemistry/fluid/. 